\title{
Theory of charge-density-wave dynamics
}

\author{
Ulrich Eckern \\ Kernforschungszentrum Karlsruhe, Institut für Nukleare Festkörperphysik, Postfach 3640, D-7500 Karlsruhe, Germany \\ Satish Ramakrishna \\ Laboratory of Atomic and Solid State Physics and Materials Science Center, Cornell University, Ithaca, New York 14853
}

(Received 15 November 1990; revised manuscript received 28 February 1991)

\begin{abstract}
Starting from the effective action for the order parameter (i.e., the generalization of the phase Hamiltonian), the generating functional governing the real-time dynamics is derived within the Keldysh formalism. As a particular advantage of this technique, it is possible to perform the average over the random (impurity) pinning potential at an early stage - this leads to an "interaction" term in the action that has peculiar time correlations. We study explicitly results in second order in the impurity-pinning potential with emphasis on the nonlinear response to a static field (the sliding mode) in two and three dimensions including the experimentally interesting crossover region. We study also the effect of thermal fluctuations for three- and two-dimensional situations and, as an example, describe how long-range Coulomb forces, which are screened by the normal electrons, can be incorporated.
\end{abstract}

\section{INTRODUCTION}

Though intensively studied for several years, both experimentally and theoretically, ${ }^{1}$ the fascinating lowtemperature transport properties of quasi-one-dimensional materials have remained a highly controversial subject. In particular, the discussion between advocates of quantum ${ }^{2}$ and classical ${ }^{3}$ concepts for an explanation of the nonlinear response is unsettled. On a very basic level, there is of course agreement that quasi-one-dimensional systems undergo a Peierls transition at low temperatures into a charge-density-wave (CDW) state, in which the equilibrium positions of the ions as well as the electron density are spatially modulated. For example, taking the CDW direction along the $x$ axis, the deviation of the electron density from its normal-state equilibrium value is given by

$$
\delta \rho=e \rho_{1} \cos [Q x+\chi(\mathbf{r}, t)]-e v_{F} N(0) \partial_{x} \chi(\mathbf{r}, t),
$$

where $Q=2 p_{F} / \hbar, \rho_{1}=2 N(0) \Delta / \hat{\lambda}$. Here $p_{F}, v_{F}, N(0), \hat{\lambda}$, and $\Delta$ denote the Fermi momentum, Fermi velocity, the normal state density of states per spin at the Fermi surface, the dimensionless electron-phonon coupling constant, and the magnitude of the order parameter, respectively. Also, the complex order parameter is given by $\Delta e^{i \chi(\mathbf{r}, t)}$ where we have anticipated that we are interested in situations where the phase of the order parameter depends upon space and time. For the current carried by the CDW, it is sufficient to consider the expression averaged over distances large compared to $Q^{-1}$, but small compared to the relevant length scale ${ }^{4}$ (see below); thus, from the continuity equation and (1), it follows that

$$
j=e v_{F} N(0) \partial_{t} \chi(\mathbf{r}, t) .
$$

In the presence of impurities, translational invariance is broken-this leads to pinning of the CDW. This phenomenon, as well as the response to a large constant field (depinning) and the response to an additional oscillating field, has been the topic of various investigations, and will also be studied in this paper.

In the next section we present a brief theoretical overview of the standard approach, ${ }^{4}$ which focuses on the phase of the order parameter as the only relevant variable, and in particular discuss the equation of motion including thermal noise. ${ }^{5}$ In the remaining sections we introduce and evaluate a different approach to the problem of pinning by impurities. The essential idea is to return to the Euclidean functional-integral formulation from which the equation of motion can be derived. Then, in a very natural way, one is led to a matrix formulation due to Keldysh, ${ }^{6}$ where the two matrix indices correspond to times being on the forward or backward branch of the contour arising in the analytic continuation to real times. As the essential feature, it turns out that the normalization of the generating functional (with the auxiliary field set to zero) is independent of the nonlinear impurity contribution. For the present problem, this means that the generating functional can be averaged easily with respect to the disorder, leading to an averaged interaction containing peculiar time correlations. In this respect, the Keldysh formulation has similarities to replica and supersymmetric techniques; in fact, the dynamic method has been applied to the problem of electron localization in random potentials. ${ }^{7} \mathrm{~A}$ similar analysis has been carried out earlier in a different area by Martin, Siggia, and Rose. ${ }^{8}$ After introducing convenient reduced variables and a condensed notation, the generating functional will be discussed in Sec. III. In Sec. IV we illustrate the approach by presenting selected results to second order in the impurity potential at zero temperature, and in particular investigate the crossover from three to two dimensions, i.e., the thickness dependence of the threshold field. ${ }^{9,10}$ In Sec. $V$ we study the effect of thermal fluctuations; the results, as it turns out, are close to those ob- 
tained by $\mathrm{Maki}^{11}$ by a completely different approach. A brief conclusion is given in Sec. VI. Finally, as a specific example, we consider the effect of long-range Coulomb forces in the Appendix.

\section{THEORETICAL OVERVIEW}

The study of the static properties of the CDW, for spatial variations that are on a much larger scale than the amplitude coherence length $\left(\xi_{A}=\hbar v_{F} / \Delta\right)$, is based on the phase Hamiltonian that includes gradient energies, the pinning energy, and the electric field. It is given by $^{4,5,12}$

$$
\begin{array}{r}
\mathscr{H}_{t}=\frac{\hbar^{2} m_{F} N(0)}{2} \int d^{3} r\left[\frac{1}{2} c^{2}\left(\partial_{x} \chi\right)^{2}+\frac{1}{2} c_{\perp}^{2}\left(\nabla_{\perp} \chi\right)^{2}\right. \\
\left.-2 e^{*} v_{F} \mathscr{E} \chi\right]+\mathscr{H}_{p},
\end{array}
$$

where the pinning energy $\mathscr{H}_{p}$ is

$$
\mathscr{H}_{p}=\int d^{3} r V_{p}(\mathbf{r}) \delta \rho / e .
$$

Here $m_{F}(\gg 1)$ denotes the Fröhlich mass relative to the band mass, $c^{2}=v_{F}^{2} / m_{F}, e^{*}=e / m_{F}, c_{\perp}$ is of the order of the phonon velocity, $\mathscr{E}$ is the electric-field component along the CDW direction, and $V_{p}(\mathbf{r})$ the random impurity potential. In (4) above, only the slowly varying (compared to $Q^{-1}$ ) contribution has to be taken into account. Restricting ourselves to the limit of weak pinning, in which an individual impurity is not strong enough to deform the CDW considerably (and thus typical length scales are large compared to the average distance between the impurities), $\mathscr{H}_{p}$ can be written as

$$
\mathscr{H}_{p}=\rho_{1} \int d^{3} r \operatorname{Re}\left[i \xi(\mathbf{r}) e^{i \chi(\mathbf{r}, t)}\right],
$$

where we defined $i \xi(\mathbf{r})=V_{p}(\mathbf{r}) e^{i Q x}$. Equation (5) follows from inserting the first part of the right-hand side (rhs) of (1) into (4); this part is related to processes with $\sim \pm Q$ momentum transfer (backscattering). The forwardscattering part, related to the second term on the rhs of (1) is linear in the phase and can be included easily (though this is not done in this paper). The complex random field $\xi(\mathbf{r})$ can be chosen to have a zero average and correlations $^{5}$ given by $\langle\xi \xi\rangle=0,\left\langle\xi^{*} \xi^{*}\right\rangle=0$, and

$$
\left\langle\xi(\mathbf{r}) \xi^{*}\left(\mathbf{r}^{\prime}\right)\right\rangle=\frac{1}{2 \pi N(0) \tau_{2}} \delta\left(\mathbf{r}-\mathbf{r}^{\prime}\right)
$$

where $\tau_{2}$ is the scattering time for processes with $\sim \pm Q$ momentum transfer.

For an investigation of the dynamics of the phase, one is led by phenomenological and other arguments ${ }^{5}$ (see also the Appendix) to the following equation of motion for $\chi(\mathbf{r}, t)$ :

$$
\frac{1}{2} \hbar^{2} m_{F} N(0)(\ddot{\chi}+\gamma \dot{\chi})=-\frac{\delta \mathcal{H}}{\delta \chi}+\zeta(\mathbf{r}, t),
$$

where $\gamma$ denotes the damping constant. The correlations of the thermal noise force $\zeta(\mathbf{r}, t)$ are given by ${ }^{5}$

$$
\left\langle\zeta(\mathbf{r}, t) \xi\left(\mathbf{r}^{\prime}, t^{\prime}\right)\right\rangle=m_{F} N(0) \gamma k_{B} T \delta\left(\mathbf{r}-\mathbf{r}^{\prime}\right) \delta\left(t-t^{\prime}\right)
$$

in accordance with the classical limit of the fluctuationdissipation theorem. Note that typically $\gamma \gg \omega_{p}$ (the pinning frequency $\omega_{p}$ is defined below), so that the overdamped limit is adequate under most circumstances. An inspection of (7) immediately leads to the characteristic scales of the problem. Consider, first, the static equation for $\mathscr{E}=0$ and for zero temperatures. In addition, let $\chi_{0}(\mathbf{r})$ be the solution of the linearized equation, i.e.,

$$
\frac{1}{2} \hbar^{2} m_{F} N(0)\left(-c^{2} \partial_{x}^{2}-c_{\perp}^{2} \nabla^{2}\right) \chi_{0}(\mathbf{r})=\rho_{1} \operatorname{Re}[\xi(\mathbf{r})] .
$$

As a result, the correlations of $\chi_{0}(\mathbf{r})$ (in three dimensions) are given by ${ }^{13}$

$$
\left\langle\left[\chi_{0}(\mathbf{r})-\chi_{0}(\mathbf{0})\right]^{2}\right\rangle=\left[\frac{x^{2}}{L_{\|}^{2}}+\frac{\mathbf{r}_{\perp}^{2}}{L_{\perp}^{2}}\right]^{1 / 2},
$$

where $L_{\perp}=L_{\|}\left(c_{\perp} / c\right) . L_{\|}$, usually called the FukuyamaLee-Rice length is easily determined from (9) and (10). Note that (10), which is a particular definition of $L_{\|}$, gives a length which differs by a numerical factor from the length obtained by minimizing the gradient plus impurity energy. ${ }^{4}$ In any case, $V_{c}=L_{\|} L_{\perp}^{2}$ represents the volume of a phase-coherent domain. The above result suggests the introduction of the dimensionless coordinates $\hat{x}=x / L_{\|}, \hat{\mathbf{r}}_{\perp}=\mathbf{r}_{\perp} / L_{\perp}$, and also a dimensionless impurity field $\hat{\xi}(\hat{\mathbf{r}})$ whose correlations are given by

$$
\left\langle\hat{\xi}(\hat{\mathbf{r}}) \hat{\xi}\left(\hat{\mathbf{r}}^{\prime}\right)\right\rangle=8 \pi \delta\left(\hat{\mathbf{r}}-\widehat{\mathbf{r}}^{\prime}\right) .
$$

This translates (9) into

$$
-\hat{\nabla}^{2} \chi_{0}=\operatorname{Re}[\hat{\xi}(\hat{\mathbf{r}})] \text {. }
$$

Similarly, we define the characteristic frequency, field and time by

$$
\omega_{p}=c / L_{\|}, \quad \mathscr{E}_{0}=\omega_{p}^{2} / 2 e^{*} v_{F}, \quad t_{0}=\gamma / \omega_{p}^{2},
$$

and put $\widehat{\mathscr{E}}=\mathscr{E} / \mathscr{E}_{0}, \hat{t}=t / t_{0}$, and $\Theta=T \gamma / \hbar \omega_{p}^{2}$. The time scaling factor is appropriate in the overdamped limit. From now on, we also omit the carets; thus (7) reduces to the following equation of motion $\left(\mu=\omega_{p}^{2} / \gamma^{2}\right)$ :

$$
\mu \ddot{\chi}+\dot{\chi}-\nabla^{2} \chi=\operatorname{Re}\left[\xi(\mathbf{r}) e^{i \chi}\right]+\mathscr{E}+\zeta(\mathbf{r}, t) .
$$

The correlations of $\zeta$ are given as

$$
\left\langle\zeta(\mathbf{r}, t) \xi\left(\mathbf{r}^{\prime}, t^{\prime}\right)\right\rangle=\frac{2 \Theta}{\lambda} \delta\left(\mathbf{r}-\mathbf{r}^{\prime}\right) \delta\left(t-t^{\prime}\right),
$$

where

$$
\lambda=\frac{1}{2} m_{F} N(0) V_{c} \hbar \gamma .
$$

Note that $\lambda$ essentially is the energy in a phase coherent volume measured in dimensionless units as constructed above. Typical parameter values are $L_{\|}$approximately equal to a few micrometers, $L_{\perp} \approx L_{\|} / 7, m_{F} \approx 100$, $\gamma \approx 10^{11} \mathrm{sec}^{-1}, \gamma / \omega_{p} \approx 100$, and thus $\lambda \approx 10^{7}$. The fact that $\lambda$ is very large allows for a classical approximation, which will be discussed below.

\section{GENERATING FUNCTIONAL}

The derivation of the equation of motion from microscopic theory has been studied in detail, ${ }^{5}$ and will only be 
sketched here. It is based on methods similar to those employed in the study of the quantum dynamics of Josephson junctions. ${ }^{14}$ In a first step, neglecting variations of the magnitude of the order parameter, which is justified in the long-wavelength low-frequency limit (compared to the amplitude coherence length $\xi_{A}$ and the gap frequency $\Delta / \hbar)$, one obtains an Euclidean action $S[\chi]$, where $\chi=\chi(\mathbf{r}, t)$ and the $t$ integration is from 0 to $-i \hbar \beta$ $(\beta=1 / T)$. The $t$ integration could also be allowed to run from $t^{*}$ to $t^{*}-i \hbar \beta$ where $t^{*}$ denotes an arbitrary (real) time. Then the integration contour is deformed such that $t$ runs from $t^{*}$ to $+\infty$, from $+\infty$ back to $t^{*}$, and finally from $t^{*}$ to $t^{*}-i \hbar \beta$. Finally, taking $t^{*} \rightarrow-\infty$ and neglecting initial correlations, one arrives at the Keldysh countour $^{6}-\infty \rightarrow \infty \rightarrow-\infty$.

Thus in a very natural way, one is led to define $\chi_{1}(\mathbf{r}, t)$ and $\chi_{2}(\mathbf{r}, t)$ on the forward and the backward branch, respectively. Neglecting for a moment the dissipative part, the real time action is found to be given by

$\mathcal{A}=\frac{\lambda}{2} \int_{-\infty}^{\infty} d t \int d^{3} r\left[\frac{\mu}{2} \dot{\chi}_{1}^{2}-\mathcal{U}\left(\chi_{1}\right)-\left(\chi_{1} \rightarrow \chi_{2}\right)\right]$,

where we have used the rescaled variables as explained above, and

$$
\begin{aligned}
& \mathcal{U}(\chi)=\frac{1}{2}(\nabla \chi)^{2}-\mathscr{E} \chi+\mathcal{U}_{p}(\chi), \\
& \mathcal{u}_{p}(\chi)=\operatorname{Re}\left[i \xi(\mathbf{r}) e^{i \chi}\right] .
\end{aligned}
$$

Generally, and in particular in the presence of dissipation which adds to (17) a contribution analogous to the single degree of freedom case ${ }^{5,15}$ (quadratic in $\chi_{1}$ and $\chi_{2}$ ), it is useful to transform to sum and difference variables. We hence define

$$
\frac{\phi_{1}}{\lambda}=\frac{\chi_{1}-\chi_{2}}{\sqrt{2}}, \quad \phi_{2}=\frac{\chi_{1}+\chi_{2}}{\sqrt{2}},
$$

where in defining $\phi_{1}$ we have anticipated that fluctuations in $\chi_{1}-\chi_{2}$ are suppressed since $\chi_{1}-\chi_{2} \simeq \lambda^{-1}$, and $\lambda \gg 1$. The quantity $\chi(\mathbf{r}, t)$ (the classical phase) discussed in Sec. II, is related to $\phi_{2}$ by $\chi=\phi_{2} / \sqrt{2}$. As a result, the action is given by

$$
\mathcal{A}=\mathcal{A}_{0}+\mathcal{A}_{p}+\mathcal{A}_{\varepsilon}
$$

where $\mathcal{A}_{0}, \mathcal{A}_{p}$, and $\mathcal{A}_{\varepsilon}$ denote the quadratic part, the pinning term, and the external field term, respectively. We find

$$
\mathcal{A}_{0}=\frac{1}{2}\left\langle\phi\left|G_{0}^{-1}\right| \phi\right\rangle
$$

using an obvious shorthand notation, e.g.,

$$
\langle A \mid B\rangle=\sum_{\alpha=1,2} \int d t \int d^{3} r A_{\alpha}(\mathbf{r}, t) B_{\alpha}(\mathbf{r}, t)
$$

and so on. The zero-order Green's function $G_{0}$ is a $2 \times 2$ matrix with the structure $e^{6,16}$

$$
G_{0}=\left[\begin{array}{cc}
0 & G_{0}^{A} \\
G_{0}^{R} & G_{0}^{K}
\end{array}\right],
$$

where $R, A$, and $K$ refer to the retarded, advanced, and
Keldysh components. Including the dissipative contribution the Fourier-transformed Green's functions are given by

$$
G_{0}^{R}(\mathbf{q}, \omega)=\left(\mu \omega^{2}+i \omega-q^{2}\right)^{-1}, \quad G_{0}^{A}=\left(G_{0}^{R}\right)^{*},
$$

and in accordance with the fluctuation-dissipation theorem,

$$
G_{0}^{K}=\frac{1}{\lambda}\left(G_{0}^{R}-G_{0}^{A}\right) \frac{2 \Theta}{\omega},
$$

where we have displayed the classical limit $\omega<<\Theta$. Quantum fluctuations can be included by the replacement $2 \Theta / \omega \rightarrow \operatorname{coth}(\omega / 2 \Theta)$. Note that the inverse has the following structure (again in the classical limit):

$$
G_{0}^{-1}=\left(\begin{array}{cc}
4 i \Theta / \lambda & \left(G_{0}^{R}\right)^{-1} \\
\left(G_{0}^{A}\right)^{-1} & 0
\end{array}\right) .
$$

Second, the impurity part is given by

$\mathcal{A}_{p}=-\lambda \int d t \int d^{3} r\left[\mathcal{U}\left(\frac{\phi_{2}+\phi_{1} / \lambda}{\sqrt{2}}\right)-\mathcal{U}\left[\frac{\phi_{2}-\phi_{1} / \lambda}{\sqrt{2}}\right]\right]$

and finally, we have

$$
\mathcal{A}_{\varepsilon}=\langle\varepsilon \mid \phi\rangle \text {, }
$$

where $\varepsilon_{1}=\sqrt{2} \mathscr{E}$ and $\varepsilon_{2}$ is an auxiliary field, to be put equal to zero after differentiation (see below). We are then left to define a generating functional to compute correlation functions with. This is

$$
Z[\varepsilon]=\mathcal{N} \int \mathcal{D} \phi_{1} \mathcal{D} \phi_{2} e^{i \mathcal{A}} .
$$

The constant $\mathcal{N}$ is chosen such that $Z\left[\varepsilon_{2}=0\right]=1$. The surprising fact is that it turns out to be independent of the nonlinear contribution $\mathcal{A}_{p}$. This important property of the Keldysh technique can be demonstrated perturbatively ${ }^{6}$ by expanding the rhs of (30) with respect to $\mathcal{A}_{p}$ and then $\mathcal{A}_{p}$ with respect to $\phi_{1}, \phi_{2}$. Taking into account the matrix structure given in (24), it follows that each contribution (for $\varepsilon_{2}=0$ ) contains a closed loop of retarded (or advanced) Green's functions following each other. However, the product of retarded (or advanced) functions in a closed loop is identically zero. This completes the proof of the above claim. As a consequence, it is straightforward to perform an impurity average at the generating functional stage itself. We define $\mathcal{A}_{\text {int }}$ as

$$
\left\langle e^{i \mathcal{A}_{p}}\right\rangle=e^{i \mathcal{A}_{\text {int }}},
$$

and taking out the zeroth-order result $\mathcal{A}_{0}[\varepsilon]$, where

$$
\mathcal{A}_{0}[\varepsilon]=\exp \left[-\frac{i}{2}\left\langle\varepsilon\left|G_{0}\right| \varepsilon\right\rangle\right)
$$

we obtain

$$
Z[\varepsilon]=Z_{0}[\varepsilon]\left\langle e^{i \mathcal{A}_{\mathrm{int}}}\right\rangle_{0},
$$

where the subscript zero indicates that the average has to be taken with the "weight" $e^{i \mathcal{A}_{0}+i \mathcal{A}_{\varepsilon}}$; it is normalized such that $\langle 1\rangle_{0}=1$. The result of the impurity average is 


$$
i \mathcal{A}_{\mathrm{int}}=-8 \pi \lambda^{2} \int d t \int d t^{\prime} \int d^{3} r \sin \frac{\phi_{1}(\mathbf{r}, t)}{\lambda \sqrt{2}} \sin \frac{\phi_{1}\left(\mathbf{r}, t^{\prime}\right)}{\lambda \sqrt{2}} \cos \left[\frac{\phi_{2}(\mathbf{r}, t)-\phi_{2}\left(\mathbf{r}, t^{\prime}\right)}{\sqrt{2}}\right) .
$$

Since $\lambda$ is large $\left(\sim 10^{7}\right)$, it is possible to approximate the sine functions to lowest order in their arguments. This leads to

$$
i \mathcal{A}_{\mathrm{int}}=-4 \pi \int d t \int d t^{\prime} \int d^{3} r \phi_{1}(\mathbf{r}, t) \phi_{1}\left(\mathbf{r}, t^{\prime}\right) \cos \left[\frac{\phi_{2}(\mathbf{r}, t)-\phi_{2}\left(\mathbf{r}, t^{\prime}\right)}{\sqrt{2}}\right] \text {. }
$$

This is a central result of this paper, on which the perturbative analysis will be based. We proceed by summarizing how physical quantities of interest are to be computed provided $Z(\varepsilon)$ or $F(\varepsilon)=-i \ln Z(\varepsilon)$ are given. For example, the average of the phase is

$$
\langle\chi(\mathbf{r}, t)\rangle=\frac{1}{\sqrt{2}}\left\langle\phi_{2}(\mathbf{r}, t)\right\rangle=\frac{1}{\sqrt{2}}\left(\frac{\delta F}{\delta \varepsilon_{2}}\right)_{\varepsilon_{2}=0} .
$$

Furthermore, the full connected Green's function is given by

$$
G^{\alpha \beta}\left(\mathbf{r}, t ; \mathbf{r}^{\prime}, t^{\prime}\right)=-\left(\frac{\delta^{2} F}{\delta \varepsilon_{\alpha}(\mathbf{r}, t) \delta \varepsilon_{\beta}\left(\mathbf{r}^{\prime}, t^{\prime}\right)}\right)_{\varepsilon 2=0},
$$

where, as we mentioned earlier $\varepsilon_{1}=\sqrt{2} \mathscr{E}$. In particular, the fluctuations of the phase are given by

$$
\left\langle\delta \chi(\mathbf{r}, t) \delta \chi\left(\mathbf{r}^{\prime}, t^{\prime}\right)\right\rangle=\frac{i}{2} G^{K}\left(\mathbf{r}, t ; \mathbf{r}^{\prime}, t^{\prime}\right),
$$

where $\delta \chi=\chi-\langle\chi\rangle$. In order to explain the general structure further, we write

$$
F=F_{0}+F_{1}, \quad F_{0}=-i \ln Z_{0}[\varepsilon]
$$

and define $|\bar{\phi}\rangle=-\hat{G}_{0}|\varepsilon\rangle$, which corresponds to free motion in the presence of an arbitrary field, and put $|\phi\rangle=|\bar{\phi}\rangle+\delta \phi$. This transformation removes the $\mathcal{A}_{\varepsilon}$ in the exponent, and we are left with the expression

$$
F_{1}=-i \ln \langle\exp (i \mathcal{A}[\bar{\phi}+\delta \phi])\rangle_{0,0},
$$

where the subscript now indicates that the "weight" is $e^{i \mathcal{A}_{0}}$. Furthermore, we consider now $F_{1}$ as a functional of $\bar{\phi}$, if convenient, and also combine all the variables into a single index $z=(\alpha, \mathbf{r}, t)$. Then we obtain immediately

$$
\begin{aligned}
-G_{0}^{-1}\left(z, z^{\prime}\right)\left\langle\phi_{z^{\prime}}\right\rangle & =-G_{0}^{-1}\left(z, z^{\prime}\right) \frac{\delta F}{\delta \varepsilon_{z^{\prime}}} \\
& =\frac{\delta F}{\delta \bar{\phi}_{z}}=\varepsilon_{z}+\frac{\delta F_{1}}{\delta \bar{\phi}_{z}},
\end{aligned}
$$

since $G_{0}\left(z, z^{\prime}\right)=G_{0}\left(z^{\prime}, z\right)$. Similarly, the Green's function is given by

$$
G\left(z, z^{\prime}\right)=G_{0}\left(z, z^{\prime}\right)+G_{0}\left(z, z_{1}\right) S\left(z_{1}, z_{2}\right) G_{0}\left(z_{2}, z^{\prime}\right)
$$

where

$$
S\left(z_{1}, z_{2}\right)=-\frac{\delta^{2} F_{1}}{\delta \bar{\phi}_{z_{1}} \delta \bar{\phi}_{z_{2}}}
$$

After differentiation, we take $\varepsilon_{2}=0$, i.e., $\bar{\phi}_{1}=0$ and $\bar{\phi}_{2}=-G_{0}^{R} \varepsilon_{1}$. Note that for constant (i.e., not varying in space and time) electric field, we have $\bar{\phi}_{2}(t)-\bar{\phi}_{2}\left(t^{\prime}\right)$ $=\varepsilon_{1}\left(t-t^{\prime}\right)=\sqrt{2} \mathscr{E}\left(t-t^{\prime}\right)$ which will be of importance later.

Finally, we remark that in first order, i.e., $F_{1} \approx F_{1}^{(1)}=\left\langle\mathcal{A}_{\text {int }}[\bar{\phi}+\delta \phi]\right\rangle_{0,0}$ this quantity is easily determined, with the result

$$
\begin{aligned}
F_{1}^{(1)}=4 \pi i \int d t \int d t^{\prime} \int d^{3} r e^{-M\left(t-t^{\prime}\right)} & {\left[\bar{\phi}_{1}(\mathbf{r}, t) \bar{\phi}_{1}\left(\mathbf{r}, t^{\prime}\right) \cos \left(\frac{\bar{\phi}_{2}(\mathbf{r}, t)-\bar{\phi}_{2}\left(\mathbf{r}, t^{\prime}\right)}{\sqrt{2}}\right)\right.} \\
& \left.-i \sqrt{2} G_{0}^{R}\left(\mathbf{0}, t-t^{\prime}\right) \bar{\phi}_{1}(\mathbf{r}, t) \sin \left(\frac{\bar{\phi}_{2}(\mathbf{r}, t)-\bar{\phi}_{2}\left(\mathbf{r}, t^{\prime}\right)}{\sqrt{2}}\right)\right],
\end{aligned}
$$

where

$$
M(t)=\frac{i}{2}\left[G_{0}^{K}(0,0)-G_{0}^{K}(0, t)\right]
$$

and we also used that $G_{0}^{R}(t=0)=G_{0}^{A}(t=0)=0$, and $G_{0}^{R}(t) G_{0}^{A}(t)=0$. Consequences of (44) will be studied below. We remark in passing that for $\varepsilon_{2}=0$, i.e., $\bar{\phi}_{1}=1$, we find that $F_{1}^{(1)}=0$, which is related to the discussion of the normalization condition given below (30).

\section{THRESHOLD FIELD AND DIMENSIONAL CROSSOVER}

In this and the following section we study the motion of the CDW in the presence of a constant external field, i.e., the static nonlinear current field characteristic. Our discussion will be based on the first-order expression for the generating functional, $F_{1}^{(1)}$ given in (44) above and thus, strictly speaking, applies only for sufficiently large $\mathscr{E}$. Nevertheless, we extrapolate the perturbative result to obtain an estimate of the threshold field. Of course, we cannot expect reliable results concerning numerical factors, but we believe that ratios and the dimensional crossover will be reasonably accurate.

Given the formal preliminaries of the previous section, the results follow easily. Consider the first component of (41) and perform the variational derivative; the result is

$$
\langle\dot{\chi}\rangle=\mathscr{E}+4 \pi \int d \tau e^{-M(\tau)} \sin (\mathscr{E} \tau) G_{0}^{R}(0, \tau) .
$$


Thermal fluctuations are related to $M(\tau)$, which will be the topic of Sec. V. Here, we restrict ourselves to $M(\tau)=0$. Clearly, the second term on the rhs of (46) represents the reduction of the CDW velocity due to impurity scattering. Neglecting the inertia, which is justified for $\mathscr{E} \ll<\mu^{-1}=\left(\gamma / \omega_{p}\right)^{2}$, we obtain the wellknown result, ${ }^{3}$ namely

$$
\langle\dot{\chi}\rangle=\mathscr{E}-4 \pi \int d \mathbf{q} \frac{\mathscr{E}}{\mathscr{E}^{2}+q^{4}}+\cdots .
$$

For three-dimensional samples, $d \mathrm{q}=d^{3} \mathrm{q} /(2 \pi)^{3}$, while for films of thickness $d<<L_{\perp}$ in the transverse direction, $d \mathrm{q}=\left(L_{\perp} / d\right) d^{2} \mathrm{q} /(2 \pi)^{2}$, and for wires of area $A<L_{\perp}^{2}$, $d \mathbf{q}=\left(L_{\perp}^{2} / A\right) d q / 2 \pi$. The integration in (47) is done easily, and we obtain the following results:

$$
\langle\dot{\chi}(\mathbf{r}, t)\rangle=\left\{\begin{array}{l}
\mathscr{E}-\sqrt{\mathscr{E} / 2}+\cdots, \quad D=3, \\
\mathscr{E}-\left(\pi L_{\perp} / 2 d\right)+\cdots, \quad D=2, \\
\mathscr{E}-\left(\pi L_{\perp}^{2} / A\right) \mathscr{E}^{-1 / 2}+\cdots, \quad D=1
\end{array}\right.
$$

The dots indicate the higher power terms, which form a power series with terms of the form $\mathscr{E}^{1-m / 2}, \mathscr{E}^{1-m}$, and $\mathscr{E}^{1-3 m / 2}$, with $m=2,3, \ldots$ from here on, for $D=3$, $D=2$, and $D=1$, respectively. Using the first-order expressions, we obtain the following estimates for the threshold field $\mathscr{E}_{T}$ from the condition $\langle\dot{\chi}\rangle=0$ :

$$
\begin{aligned}
& \mathscr{E}_{T}^{3 D}=\frac{1}{2} \\
& \mathscr{E}_{T}^{2 D}=\left(\pi L_{\perp} / 2 d\right) \\
& \mathscr{E}_{T}^{1 D}=\left(\pi L_{\perp}^{2} / A\right)^{2 / 3} .
\end{aligned}
$$

In particular, returning to physical units, we find the ratio ${ }^{10,9}$

$$
\frac{\left(\mathscr{E}_{T}^{2 D} d\right)^{2}}{\mathscr{E}_{T}^{3 D}}=\frac{\pi^{2}}{4}\left(\frac{L_{\perp}}{L_{\|}}\right)^{2} \frac{\hbar v_{F}}{e} .
$$

Note that this quantity is about a factor of 2 smaller than the estimate that Bardeen ${ }^{9}$ obtains based on his quantum theory. The ratio is also in good accord with recent experiments if we choose $L_{\perp} / L_{\|} \approx 1 / 3$. In the experiment, in fact, the thickness dependence of the threshold field has been investigated. In order to study this crossover, we put

$$
\int d \mathbf{q}=\frac{L_{\perp}}{d} \sum_{n} \int \frac{d^{2} q}{(2 \pi)^{2}}
$$

where the sum is over the discrete states in the transverse direction, with the result

$$
\dot{\chi}(\mathbf{r}, t)=\mathscr{E}-\frac{L_{\perp}}{d} \sum_{n}\left[\frac{\pi}{2}-\arctan \left[\frac{q_{n}^{2}}{\mathscr{E}}\right]\right],
$$

where $q_{n}=2 \pi n L_{\perp} / d$. Note that actually the two-

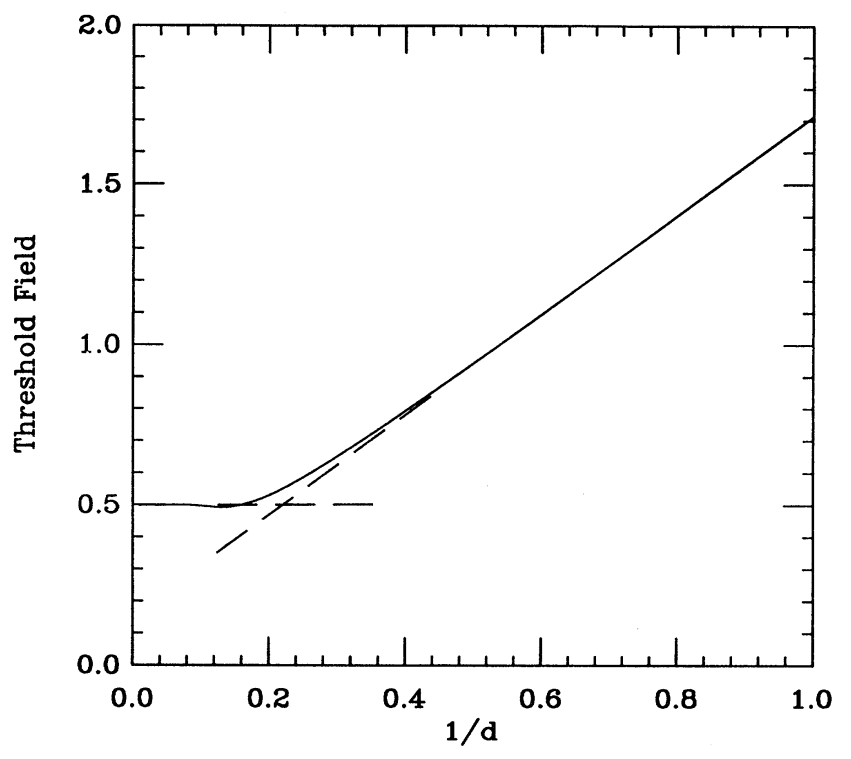

FIG. 1. Threshold field vs thickness for zero temperature as obtained numerically. The quantities are given in dimensionless units as mentioned in (11) and (13). The dashed lines indicate the asymptotic behavior for $d \gg L_{\perp}$ and $d \leq L_{1}$, as given by (51) and (57).

dimensional result given in (49) applies only for $d<<L_{\perp} / \mathscr{E}^{1 / 2}$, and thus even thin films show a crossover to three-dimensional behavior for sufficiently large fields. Putting again $\langle\dot{\chi}\rangle=0$ and expanding (56) for small $d$, we find

$$
\mathscr{E}_{T}=\frac{\pi L_{\perp}}{2 d}+\frac{\pi}{24}+\cdots, d \leq L_{\perp} .
$$

Equating this expression with $\mathscr{E}_{T}^{3 D}=\frac{1}{2}$, we find that the crossover occurs at the film thickness $d^{*} / L_{\perp} \approx 4.3$, which is easily confirmed by a numerical evaluation of the sum in (56) (see Fig. 1). An excellent approximation for $\mathscr{E}_{T}(d)$ is $\mathscr{E}_{T} \approx \frac{1}{2}$ for $d>d^{*}$ and $\mathscr{E}_{T}(d)$ as given in (57) for $d<d^{*}$. Thus given a measured crossover thickness $d^{*}$ and the ratio (54), it is possible to infer the characteristic lengths. Note that the experiment also confirms that the pinning in $\mathrm{NbSe}_{3}$ doped with $\mathrm{Ta}$ is weak. ${ }^{10}$

\section{THERMAL FLUCTUATIONS}

Thermal fluctuations enter (46) through $M(\tau)$, which is related to the time dependence of phase fluctuations at the same point in space. From (45) and (38), we obtain

$$
M(\tau)=\frac{1}{2}\left\langle[\delta \chi(0, \tau)-\delta \chi(0,0)]^{2}\right\rangle .
$$

Thus $M(\tau=0)=0$ and $M(\tau) \geq 0$ for all times. Explicitly, using the results (25) and (26), this quantity is given by

$$
M(\tau)=\frac{2 \Theta}{\lambda} \int \frac{d \omega}{2 \pi} \int d \mathbf{q} \frac{1-e^{-i \omega \tau}}{\left(\mu \omega^{2}-q^{2}\right)^{2}+\omega^{2}}
$$

in the classical limit. (Note that in the quantum limit $\Theta \rightarrow 0$, the integrand has to be multiplied by $|\omega| / 2 \Theta$.) 
Clearly, (59) requires a short-wavelength cutoff, which we introduce into this expression by multiplying by $\exp \left(-\tau_{0} q^{2}\right)$. Physically, this can be motivated by the observation that the long-wavelength expansion, on which all our results are based, ceases to be valid on the scale of the amplitude coherence length. Thus, $\tau_{0} \sim\left(\xi_{A} / L_{\|}\right)^{2}$ $\sim 10^{-4}$ in order of magnitude. Neglecting for simplicity the inertia, we obtain

$$
M(\tau)=\frac{\Theta}{\lambda} \frac{1}{4 \pi^{3 / 2}}\left(\frac{1}{\sqrt{\tau_{0}}}-\frac{1}{\sqrt{\tau+\tau_{0}}}\right) .
$$

Thus $M(\tau)$ increases initially on the scale given by $\tau_{0}$, and approaches for $\tau \gg \tau_{0}$ the value

$$
M_{\infty}=\left\langle[\delta \chi(0,0)]^{2}\right\rangle=\frac{\Theta / \lambda}{4 \pi^{3 / 2} \sqrt{\tau_{0}}}=\frac{2 T}{T_{0}},
$$

where the last equality in this relation defines the characteristic temperature ${ }^{17}$ (and is chosen so that it agrees with Ref. 11). Note that (61) is at best an order-of-magnitude estimate for $T_{0}$ which should be determined actually by comparison with experiment.

Inserting (61) into (46), however, we realize that the time scale in that expression is set by $\mathscr{E}^{-1}$. Thus, except for extremely large fields, we conclude that it is a sufficient approximation to use

$$
e^{-M(\tau)} \approx e^{-M_{\infty}}=e^{-2 T / T_{0}} .
$$

We then immediately deduce the temperature dependence of the threshold field, which by comparison with $(48)-(50)$ is given by

$$
\frac{\mathscr{E}_{T}(T)}{\mathscr{E}_{T}(0)}=\left(e^{-T / T_{0}}\right)^{4 /(4-D)},
$$

in agreement with Ref. 11 . The agreement of the static considerations of that paper with our investigation of the sliding mode is related to the fact that fast and short wavelength fluctuations dominate the behavior; for these, even a "slowly" moving CDW is a static object to a good approximation. As another consequence, we note that the three-dimensional expression for the fluctuations is adequate as long as $d \gg \xi_{A}$. Presently, however, it remains doubtful whether the observed temperature dependence of the threshold field (for low temperatures) can be explained in all cases by the above arguments. ${ }^{18}$

Finally, we remark that for a film of thickness $d \leq L_{\perp}$ [compare (57)], the temperature dependence of the threshold field is given by

$$
\mathscr{E}_{T}(T)=\frac{\pi L_{\perp}}{2 d} e^{-2 T / T_{0}}+\frac{\pi}{24} e^{-4 T / T_{0}}+\cdots, d \leq L_{\perp} .
$$

As a consequence, the crossover thickness $d^{*}$ is also temperature dependent, with the result

$$
d^{*}(T) / d^{*}(T=0)=\exp \left(2 T / T_{0}\right) .
$$

Thus $d^{*}$ increases with increasing temperature, which reflects the temperature dependence of the FukuyamaLee-Rice length.

\section{CONCLUSIONS}

In summary, we introduced in this paper a different approach to the random disorder inherent in the charge density wave problem. Within this technique, it was possible to derive an impurity averaged generating functional on which, as we hope, further nonperturbative studies can be based. Presently, however, we have not been successful with this goal. Clearly, within perturbation theory which we have studied in some detail in order to illustrate some features of our method, we obtained results which agree with standard perturbation theory. In particular, we studied the dimensional crossover in the threshold field; the results are in reasonable agreement with experiment ${ }^{10}$ (but this is also true for Bardeen's quantum theory ${ }^{9}$ ). In addition, our results for the temperature dependence agree with an earlier theoretical approach $^{11}$ which is based on completely different arguments.

\section{ACKNOWLEDGMENTS}

U.E. thanks Professor V. Ambegaokar for his hospitality during a visit at Cornell University, where this collaboration was initiated, and the Deutsche Forschungsgemeinschaft for support. S.R. wishes to thank Professor V. Ambegaokar for comments, criticism, and encouragement, the Institut für Nukleare Festkörperphysik, Kernforschungszentrum Karlsruhe and the Institut für Theorie der Kondensierten Materie, Universität Karlsruhe for their hospitality, and the Cornell University Materials Science Center for financial support through Grant No. DMR-8818558-A02. We also acknowledge useful discussions with Professor R. Thorne and $\mathbf{J}$. McCarten.

\section{APPENDIX}

In order not to interrupt our line of arguments, we have excluded in the above sections a detailed discussion of the microscopic theory that leads to (3) including the dissipation and fluctuating force. There is, however, a rather important contribution arising from the longrange Coulomb forces. These, as is well known, are screened by the normal electrons (see Sneddon ${ }^{3}$ and also Refs. 12 and 19). This aspect deserves further attention and will be discussed in this appendix.

Though we could base our arguments again on the path integral version ${ }^{5}$ of the microscopic theory, including the Coulomb interaction, the result can be summarized in the low-temperature limit very simply as follows. Note that for low temperature, screening is entirely due to the normal electrons, i.e., those from parts of the Fermi surface not participating in the CDW transition. Thus define

$$
V(\mathbf{q}, \omega)=\frac{V_{0}(\mathbf{q})}{\epsilon_{n}(\mathbf{q}, \omega)}, \quad V_{0}(\mathbf{q})=\frac{4 \pi e^{2}}{q^{2}}
$$

which is the Coulomb interaction screened by the normal electrons (at Matsubara frequency $\omega$ ). The dielectric function $\epsilon_{n}(\mathbf{q}, \omega)$ is related to the density-density correla- 
tion function of the normal electrons in the usual way. As a result, the Coulomb contribution to the Euclidean action $\mathscr{\rho}_{C}$ is given by $\left(\hbar=k_{B}=1\right)$ :

$$
\mathcal{S}_{C}[\chi]=\frac{1}{2} \int d x d x^{\prime} \frac{\delta \rho(x)}{e} V\left(x-x^{\prime}\right) \frac{\delta \rho\left(x^{\prime}\right)}{e},
$$

where $x=(\mathbf{r}, \tau)$, the $\tau$ integration is between 0 and $\beta=1 / T$ and $\delta \rho$ is given by expression (1). Presently, we concentrate upon the first term in (1) (however, see Ref. 5) and use the long-wavelength, low-frequency limit of the dielectric function

$$
\epsilon(\mathbf{q}, \omega)=1+\left(\frac{q_{T F}}{1}\right)^{2} \frac{\mathbf{q} \hat{D} \mathbf{q}}{|\omega|+\mathbf{q} \hat{D} \mathbf{q}} .
$$

Here $q_{T F}^{2}=8 \pi e^{2} N_{n}(0), N_{n}(0)$ is the normal electron density of states at the Fermi surface, and $\mathbf{q} \widehat{D} \mathbf{q}=q_{x}^{2} D_{\|}$ $+q_{1}^{2} D_{\perp}$, where $D_{\|}$and $D_{\perp}$ are the parallel and perpendicular diffusion constants respectively. Actually, the " 1 " in (A3) is negligible. Taking all this into account, we find in the Fourier representation

$\rho_{C}[\chi]=\frac{v_{F}^{2} N(0)^{2}}{4 N_{n}(0)} T \sum_{\omega_{n}} \int \frac{d \mathbf{q}}{(2 \pi)^{3}} q_{x}^{2}\left|\chi_{\mathbf{q}, \omega}\right|^{2}\left(1+\frac{|\omega|}{\mathbf{q} \hat{D} \mathbf{q}}\right)$.

Comparison with the Hamiltonian in (3) shows that the first term in (A4) implies simply a renormalization of the phason speed, according to

$$
c^{2} \rightarrow c^{2}\left[1+\frac{N(0)}{N_{n}(0)}\right]
$$

which can be a considerable enhancement provided large parts of the Fermi surface are affected by the CDW transition $\left[N(0) \gg N_{n}(0)\right]$. On the other hand, we write the second term as follows:

$$
\rho_{C}=\frac{m_{F} N(0)}{4} T \sum_{\omega_{n}} \int \frac{d^{3} \mathbf{q}}{(2 \pi)^{3}}|\omega|\left|\chi_{\mathbf{q}, \omega}\right|^{2} \gamma_{c}(\mathbf{q})
$$

where we defined

$$
\gamma_{c}(\mathbf{q})=\frac{v_{F}^{2} N(0)}{m_{F} N_{n}(0)} \frac{q_{x}^{2}}{\mathbf{q} \hat{D} \mathbf{q}} .
$$

Note that (A5) and (A7) have also been obtained in Ref. 12 , where the more general case of finite temperatures was considered.

The following steps of analytic continuation are now straightforward, as described in Sec. III. Thus we find the trivial change in $\mathscr{H}$, as given by (A5), but in addition the dissipative term due to $\gamma_{c}$ becomes nonlocal in space according to [compare (7)]

$$
\gamma \dot{\chi} \rightarrow \int d^{3} \mathbf{r}^{\prime} \gamma\left(\mathbf{r}-\mathbf{r}^{\prime}\right) \dot{\chi}\left(\mathbf{r}^{\prime}, t\right),
$$

where $\gamma(\mathbf{r})=\gamma_{0} \delta(\mathbf{r})+\gamma_{c}(\mathbf{r})$. Furthermore, the correlations of the fluctuating force are modified accordingly, with the result

$$
\gamma \delta\left(\mathbf{r}-\mathbf{r}^{\prime}\right) \rightarrow \gamma\left(\mathbf{r}-\mathbf{r}^{\prime}\right)
$$

This is highly anisotropic, provided $\gamma_{c}$ dominates. An important feature of the Coulomb contribution is expressed by the statement that

$$
\int d \mathbf{r} \gamma_{c}(\mathbf{r})=0 \text {. }
$$

In order to discuss the consequences of the Coulomb contribution to our results, we introduce reduced quantities as described in Sec. II, using presently the renormalized phason speed [Eq. (A5)] and the bare viscosity $\gamma_{0}$ to effect the normalizations. In particular, (A7) can be written as

$$
\gamma_{c}(\mathbf{q})=\gamma_{c 0} f(\mathbf{q})
$$

where $\gamma_{c 0}=v_{F}^{2} N(0) / m_{F} N_{n}(0) \widetilde{D}_{\|}$and

$$
f(\mathbf{q})=\frac{q_{x}^{2} \widetilde{D}_{\|}}{\mathbf{q} \widehat{\tilde{D}} \mathbf{q}},
$$

where $\widetilde{D}_{\|}=D_{\|} / L_{\|}^{2}$ and $\widetilde{D}_{\perp}=D_{\perp} / L_{\perp}^{2}$. The general discussion of Sec. III still applies, except for a redefinition of the zero-order Green's function $G_{0}$, which is given by

$$
G_{0}^{R}(\mathbf{q}, \omega)=\left\{i \omega\left[1+\gamma_{c}(\mathbf{q}) / \gamma_{0}\right]-q^{2}\right\}^{-1}
$$

in comparison with (25); the inertia term is neglected immediately. In addition, we have in (27),

$$
\Theta \rightarrow \Theta\left[1+\gamma_{c}(\mathbf{q}) / \gamma_{0}\right],
$$

which implies that the fluctuation-dissipation theorem remains unchanged.

Turning now to the explicit results of Secs. IV and V, we consider again (41) within the approximation (44). Due to the property (A10), the leading term in (47), which represents a uniformly sliding $\mathrm{CDW}$, is unchanged. This implies that the high field conductivity is given by the bare viscosity. ${ }^{12,3,19}$ While the correction term is affected, it follows nevertheless that it is formally still given by (46); explicitly, the second term on the rhs of (47) is now modified according to

$$
\mathscr{E} \rightarrow \mathscr{E}\left[1+\gamma_{c}(\mathbf{q}) / \gamma_{0}\right]
$$

Note that $\gamma_{c}(\mathbf{q})$ depends only upon the direction of the wave vector; hence we obtain as in (48)-(50)

$$
\langle\dot{\chi}\rangle=\mathscr{E}-C_{D} \mathscr{E}^{D / 2-1},
$$

where for wires $(D=1)$ and films $(D=2), C_{D}$ equals the values given in (49) and (50). On the other hand, for $D=3$,

$$
C_{3}=\left\langle\left[\frac{1}{2}\left[1+\frac{\gamma_{c}(\mathbf{q})}{\gamma_{0}}\right]\right]^{1 / 2}\right\rangle_{\Omega},
$$

where the angular brackets denote an angular average. At low temperatures, we expect $\gamma_{0}$ to become small; hence it is sensible to consider a limit $\gamma_{c 0} \gg \gamma_{0}$, in which case

$$
C_{3}=\left(\frac{\gamma_{c 0}}{\gamma_{0}}\right)^{1 / 2}\left\langle\left(\frac{f(\mathrm{q})}{2}\right)^{1 / 2}\right\rangle_{\Omega} .
$$


This expression is a decreasing function of $x=D_{1} L_{\|}^{2} /$ $D_{\|} L_{1}^{2}$, with $C_{3}(0)=\left(\gamma_{c 0} / 2 \gamma_{0}\right)^{1 / 2}, C_{3}(1)=C_{3}(0) / 2$, and

$$
C_{3}(x>1) \approx C_{3}(0) x^{-1 / 2} .
$$

Thus the enhancement of $C_{3}$ due to the prefactor in (A18) is partly compensated for $x \gg 1$, which seems to be the relevant limit. These results imply an enhancement of $\mathscr{E}_{T}^{3 D}$ over the value (51), provided $C_{3}>1 / \sqrt{2}$.

Finally, considering thermal fluctuations (Sec. V), the approximation $M(\tau) \approx M_{\infty}$ is adequate; in addition this quantity is not modified compared to (61). This is because it characterizes the equal-time correlations of the phase.
${ }^{1}$ Reviews can be found in Electronic Properties of Inorganic Quasi-One Dimensional Compounds, edited by P. Monceau (Reidel, Dordrecht, 1985).

2J. Bardeen, Phys. Scr. T 27, 136 (1989); see also further references therein.

${ }^{3}$ L. Sneddon, M. C. Cross, and D. S. Fisher, Phys. Rev. Lett. 49, 293 (1982); L. Sneddon, Phys. Rev. B 29, 719 (1984); D. S. Fisher, Phys. Rev. Lett. 50, 1486 (1983); S. N. Coppersmith and P. B. Littlewood, ibid. 57, 1927 (1986).

${ }^{4}$ H. Fukuyama and P. A. Lee, Phys. Rev. B 17, 535 (1978); P. A. Lee and T. M. Rice, ibid. 19, 3970 (1979).

${ }^{5}$ U. Eckern and A. Geier, Z. Phys. B 65, 15 (1986).

${ }^{6}$ L. V. Keldysh, Zh. Eksp. Teor. Fiz. 47, 1515 (1964) [Sov. Phys. JETP 20, 1018 (1965)]; for a brief review of this technique, and applications to a single degree of freedom, see also $U$. Eckern and F. Pelzer, Europhys. Lett. 3, 131 (1987).

${ }^{7}$ R. Kree, Z. Phys. B 65, 505 (1987).

${ }^{8}$ P. C. Martin, E. Siggia, and H. Rose, Phys. Rev. A 8, 423 (1973).

${ }^{9}$ J. Bardeen, Phys. Rev. Lett. 64, 2297 (1990).
${ }^{10} \mathrm{~J}$. McCarten, M. Maher, T. L. Adelman, and R. E. Thorne, Phys. Rev. Lett. 63, 2841 (1989).

${ }^{11}$ K. Maki, Phys. Rev. B 33, 2852 (1986); K. Maki and A. Virosztek, ibid. 39, 9640 (1989).

${ }^{12}$ U. Eckern, Z. Phys. B 74, 413 (1989).

${ }^{13}$ K. B. Efetov and A. I. Larkin, Zh. Eksp. Teor. Fiz. 72, 2350 (1977) [Sov. Phys. JETP 45, 1236 (1977)].

${ }^{14}$ U. Eckern, G. Schön, and V. Ambegaokar, Phys. Rev. B 30, 6419 (1984).

${ }^{15}$ A. O. Caldeira and A. J. Leggett, Phys. Rev. Lett. 46, 211 (1981); Ann. Phys. (N.Y.) 149, 374 (1983).

${ }^{16}$ Though other forms are possible, this choice has been found to be convenient.

${ }^{17}$ In physical units, the characteristic temperature is given by $k_{B} T_{0} \sim m_{F} N(0) V_{c}\left(\hbar \omega_{p}\right)^{2}\left(\xi_{A} / L_{\|}\right)$; note that this expression is independent of the damping constant $\gamma$.

${ }^{18}$ R. E. Thorne (private communication).

${ }^{19}$ U. Eckern, J. Low Temp. Phys. 62, 525 (1986).

${ }^{20}$ P. B. Littlewood, Phys. Rev. B 36, 3108 (1987); T. Baier and W. Wonneberger, Z. Phys. B 79, 211 (1990). 